the barriers. Primitive electron pumps have existed since 1990, but this is the first time that changes in charge have been detected for each hop of an electron.

The pump transferred just a few dozen electrons per second - slow enough to permit precision measurement and thus to provide proof of principle for redefining the amp. But this is only a first step: the set-up would not be practical for calibrating current-measuring ammeters, which need to run at higher currents. The ultimate goal is to create a 'mise en pratique' - a standard-setting experiment that can be reproduced in any lab to calibrate measurements of current precisely - so the race

"It's
impossible to
say which is
the winning
concept."
is now on to combine Schumacher's validation method with a higher-current pump.

In 2012, Giblin pioneered a semi-

conductor single-electron pump that transferred nearly one billion electrons per second (ref. 2), but he could not track them one by one. Other types of pump include turnstiles, in which electrons tunnel between superconducting wires, and tunnel junctions, in which electrons tunnel between aluminium islands separated by layers of insulating oxide. But these must also be operated at relatively low currents to track single electrons. "It's impossible to say which is the winning concept," says Jukka Pekola, a physicist at Aalto University in Espoo, Finland, who reviewed approaches to redefining the amp in 2013 (ref. 3).

Still, the amp is in good shape for the November meeting, as is the kelvin. The charge of the electron and Boltzmann's constant have both been measured precisely, so both units are ready to be redefined today, says François Piquemal, a physicist at the National Laboratory of Metrology and Testing in Paris.

But the process could be delayed until the 2018 meeting of the BIPM general conference. All four units are intertwined, so the plan is to redefine them all at once, and the kilogram is causing problems. There are two rival approaches to its redefinition: a watt balance, which would balance a test mass against Earth's gravity in terms of electrical power, and a precise count of atoms in a sphere of silicon. The two approaches give slightly different answers. Piquemal says that the differing approaches need to be reconciled before the units can be redefined.

\footnotetext{
1. Fricke, L. et al. Preprint at http://arxiv.org/ abs/1312.5669 (2013).

2. Giblin, S. P. et al. Nature Commun. 3,930 (2012).

3. Pekola, J. P. et al. Rev. Mod. Phys. 85, 14211472 (2013)
}

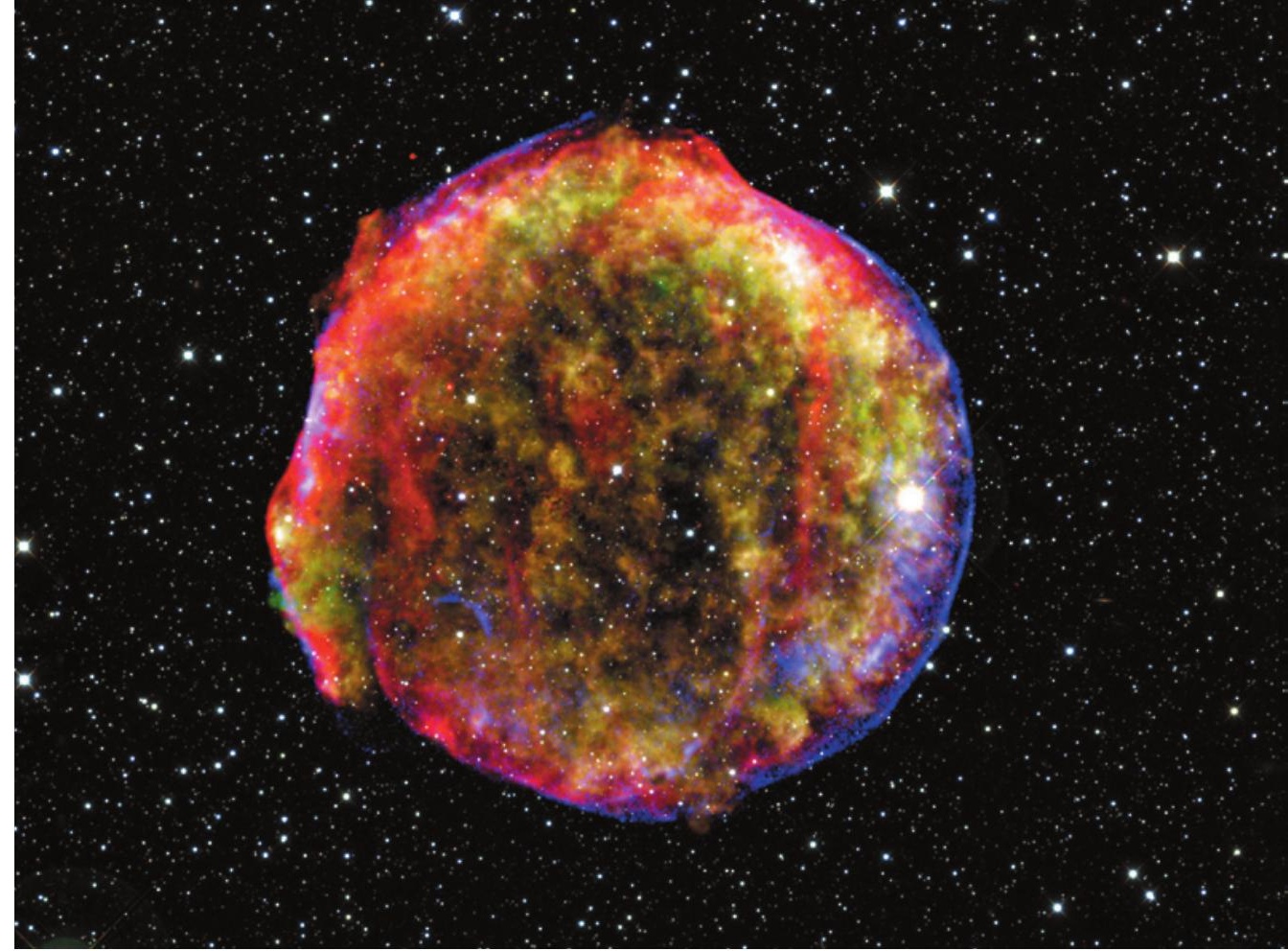

This remnant of Tycho's supernova was created by a type la supernova in 1572 .

\title{
ASTROPHYSICS
}

\section{Kepler clue to \\ supernova puzzle}

\section{Two white dwarfs favoured as precursors of type Ia supernovae.}

\section{BY RON COWEN}

$\mathrm{T}$ They are cosmic detonations that briefly outshine the light of entire galaxies. And they were a crucial tool in the discovery of dark energy, the force that is accelerating the expansion of the Universe. Yet the process that gives rise to type Ia supernovae has remained mysterious.

Now, light from two of these stellar explosions has been captured in finer temporal detail than ever before, and the data are adding weight to an emerging view: that the explosions result from the merger of two white dwarfs, the burnt-out, Earth-sized remnants of Sun-like stars. The finding erodes a longstanding view that type Ia supernovae arise from a single white dwarf accruing material from an ordinary companion star, either a Sunlike star or an elderly, bloated red giant.

The data have come from an unlikely source: NASA's Kepler mission, the space telescope that searched for alien planets by staring at some 150,000 stars in nearby reaches of the Milky Way. Distant galaxies also lurk in the telescope's field of view, and its ability to collect data every 30 minutes, along with its sensitivity to tiny changes in brightness, made it ideal for recording the rise and fall of light emitted during supernovae.

Robert Olling, an astronomer at the University of Maryland in College Park, was lucky enough to find two type Ia supernovae after a two-year survey of some 400 galaxies in Kepler's field. He reported them on 8 January at a meet ing of the American Astronomical Society near Washington DC. "As a technical tour de force, it's really cool to use Kepler for more than it was intended," says Robert Kirshner, an astronome at the Harvard-Smithsonian Center for Astrophysics in Cambridge, Massachusetts.

The exceptional smoothness of the Kepler data is helping researchers to distinguish between the two competing explosion scenarios. Both require that a white dwarf takes on material from a companion object until the pressure ignites a runaway thermonuclear explosion. But in the ordinary com panion model, the expanding shell of material from the white dwarf would ram into the normal star, generating extra heat and light that would show up as a bump in the first days of a supernova's brightening. No such bump exists in Olling's data.

That essentially rules out all red giant companions, says Olling, because these larger stars would result in a sizeable bump. But the data could still be compatible with smaller, more 


\section{A STAR'S LAST RITES}

Light captured from type la supernovae supports the idea that merging white dwarfs lead to the explosions.

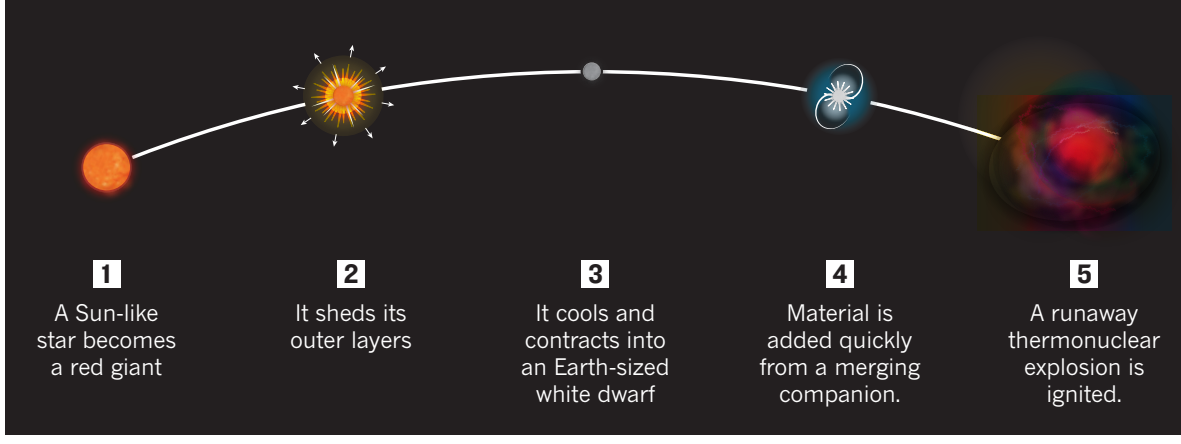

Sun-like companions, says Daniel Kasen, an astronomer at the University of California, Berkeley, and a collaborator on the survey. Not only would these stars cause a much smaller bump, but the bump could be missed completely depending on the observer's viewing angle, he says. If the supernova lay between Kepler and the companion star, for example, the associated bump would probably not be seen.

For years, the idea that type Ia supernovae might arise from merging white dwarfs was discounted because the final stages of the merger were thought to occur slowly, over the course of thousands of years. Such a slow accretion of material would be more likely to lead to the formation of a neutron star. Then, from around 2010, simulations began to suggest that the mergers could occur in seconds or minutes, allowing for the sudden pressure change that results in an explosion, says Stan Woosley, a theorist at the University of California, Santa Cruz (see 'A star's last rites').

Craig Wheeler, a supernova theorist at the University of Texas at Austin, says that there are still problems with the merger model. For example, he says, simulations of the mergers often produce highly asymmetric explosions, yet observations so far tend to be more spherical. And spectroscopic observations - which split light into its component wavelengths - have not found as much radiation from ionized iron atoms as merger simulations predict.

Kepler's exoplanet-hunting days ended in May 2013 after mechanical failures prevented it from pointing precisely enough for that task. But Olling says that the craft could continue to hunt for type Ia supernovae because the bright explosions do not require precise pointing.

It will be crucial to make simultaneous observations from ground-based telescopes, he notes, because Kepler only records brightness and cannot split light into spectra. But to perform such joint observations, Kepler will need to point in the opposite direction; Olling hopes that the Kepler team will agree to this. NASA is expected to announce its plans for the impaired spacecraft this summer.

\section{CORRECTION}

This News story 'Particle-physics papers set free' (Nature 505, 141; 2014) wrongly stated that CERN has decreed that all articles based on its research must be open access. In fact, it is still reviewing its policy. And in the World View 'Academics should not remain silent on hacking' (Nature 504, 333; 2013), the URL for the non-profit organization recruiting experts should have been opencryptoaudit.org. 